\title{
Effects of a tailored lifestyle self-management intervention (TALENT) study on weight reduction: a randomized controlled trial
}

This article was published in the following Dove Press journal:

Diabetes, Metabolic Syndrome and Obesity:Targets and Therapy

19 June 2017

Number of times this article has been viewed

\author{
Dieter Melchart ${ }^{1,2}$ \\ Peter Löw ${ }^{3}$ \\ Erich Wühr ${ }^{4}$ \\ Victoria Kehl ${ }^{5}$ \\ Wolfgang Weidenhammer' \\ 'Competence Center for \\ Complementary Medicine and \\ Naturopathy, Klinikum Rechts der \\ Isar, Technische Universität München, \\ Munich, Germany; ${ }^{2}$ Institute for \\ Complementary and Integrative \\ Medicine, University Hospital \\ Zurich, University of Zurich, Zurich, \\ Switzerland; ${ }^{3}$ Medical Practice for \\ Internal Medicine, Treuchtlingen, \\ ${ }^{4}$ Faculty for Applied Health Care \\ Science, Deggendorf Institute of \\ Technology, Deggendorf, Institute for \\ Medical Statistics and Epidemiology, \\ Munich Trial Centre, Klinikum Rechts \\ der Isar, Technische Universität \\ München, Munich, Germany
}

Correspondence: Wolfgang

Weidenhammer

Competence Center for Complementary Medicine and Naturopathy, Klinikum Rechts der Isar, Technische Universität München, 9 Kaiserstrasse, Munich 8080I, Germany

Tel +4989726 6970

Email wolfgang.weidenhammer@tum.de
Objective: Overweight and obesity are globally increasing risk factors for diseases in the context of metabolic syndrome. A randomized controlled trial was conducted to investigate whether there are any existing differences between two lifestyle intervention strategies with respect to weight reduction after 1 year.

Subjects and methods: A total of 166 subjects with a body mass index of $28-35 \mathrm{~kg} / \mathrm{m}^{2}$ were enrolled in this trial at seven study centers; 109 were randomly allocated to the intervention group (comprehensive lifestyle modification program: web-based Individual Health Management [IHM]) with 3-month reduction phase plus 9-month maintenance phase, and 57 were allocated to the control group (written information with advice for healthy food habits: usual care [UC]). Body weight, waist circumference, blood pressure, laboratory findings, and bioimpedance analysis used to determine body composition were measured at baseline and after 3, 6, 9, and 12 months. The primary outcome parameter was body weight at month 12 compared to baseline. Results: With respect to baseline status there were no statistically significant differences between the groups. Based on the intent-to-treat population, body weight showed a mean decrease of $8.7 \mathrm{~kg}$ (SD 6.1) in the intervention group (IHM) and $4.2 \mathrm{~kg}$ (SD 5) in the control group (UC) at month 12 . This statistically significant difference $(P<0.001)$ was confirmed by various sensitivity analyses. Body mass index, waist circumference, high-density lipid cholesterol, body fat, and the ratio of fat and body cell mass improved to a significantly higher degree in the IHM group. Conclusion: IHM proved to be superior to UC in weight reduction after 1 year. With a mean loss of about $10 \%$ of the baseline weight, a clinically high relevant risk reduction for cardiometabolic diseases is achievable.

Keywords: Individual Health Management, IHM, overweight, obesity, prevention, lifestyle modification

\section{Background}

Overweight and obesity are a rising challenge for health care worldwide. Between 1975 and 2014 there was an increase in the global age-standardized mean body mass index (BMI) in men from 21.7 to 24.2 and in women from 22.1 to $24.4 .^{1}$ In developed countries such as the US, the figures are markedly higher. Approximately two-thirds of adults are overweight (BMI of 25-29.9) or obese (BMI $\geq 30$ ). ${ }^{2,3}$

Obesity is associated with many comorbid conditions that have major implications on longevity, quality of life, and health-care costs. ${ }^{4,5}$ It could be shown that a strong and linear association exists between BMI (>20) and the risk of developing type 2 diabetes, hypertension, cardiovascular disease, and other chronic diseases in both men 
and women. ${ }^{6}$ A very recent analysis revealed for European countries that even in moderate forms of obesity, all-cause mortality was distinctly elevated: BMI 27.5-30 (hazard ratio 1.21) and BMI 30-35 (hazard ratio 1.52). ${ }^{3}$ Obesity has been proposed by the American College of Cardiology/American Heart Association and Task Force on Practice Guidelines to be regarded as a "disease" requiring treatment."

Prevention and treatment of obesity is a multidisciplinary approach involving comprehensive strategies of psychotherapeutic elements, different types of diet resulting in an energy deficit, and behavior modification supporting changes in diet and exercise in everyday life. ${ }^{8,9}$ Enhanced lifestyle counseling has proved to be superior to usual care with respect to weight reduction. ${ }^{10}$ Compared to minimal or no intervention, it can be shown that self-management plus exercise prevented weight increase in patients with cardiometabolic disease. ${ }^{11}$ Although there is a wide range of different popular commercial diets available, weight-loss programs having their efficacy scientifically evaluated are still rare. ${ }^{12,13}$ Further, long-term adherence to such lifestyle changes is usually low. ${ }^{14}$

We developed an intervention program called Individual Health Management (IHM), which is designed to promote changes across multiple health behaviors, simultaneously aiming for participation of healthy and ill people, in order to enhance individual responsibility, self-determination, and health literacy. Self-management supporting the participants to optimize physiological skills and psychosocial competencies is considered the core element of the intervention. It comprises areas such as physical activity, nutrition, self-efficacy, and social support, and follows an educational concept of blended learning. ${ }^{15}$

The primary objective of this study was to examine whether the IHM intervention strategy was superior to a control condition (usual care [UC]) with respect to weight reduction at month 12 in overweight persons (BMI 25-30) or those with grade 1 obesity (BMI 30-35). Since a BMI of 25-30 is not consistently associated with increased mortality, ${ }^{4}$ we set the lower threshold for BMI to 28 , resulting in a target range of 28-35. Further objectives of the tailored lifestyle self-management intervention (TALENT) study are the comparative analysis of secondary outcome parameters such as BMI, waist circumference, and laboratory findings.

\section{Subjects and methods Study design}

This study was a multicenter randomized controlled trial comprising six study centers located in different health resorts all over Bavaria, and complemented by the outpatient unit of the Competence Centre for Complementary Medicine and Naturopathy at Klinikum Rechts der Isar, Munich. The study centers were affiliated to local private practices of general practitioners, which are pooled into a centrally coordinated network of health promotion called IHM-Campus. Study duration for each participant was 12 months, with five sequential examinations at equal intervals of 3 months (baseline at month 0 , and after 3, 6, 9, and 12 months). The study was supervised by a professional institution with acknowledged expertise in supervision of clinical trials of all kinds (Munich Study Centre, Technical University of Munich), including central indoor monitoring and on-site visits at the participating study centers.

The study was registered in advance at German Clinical Trials Register Freiburg (DRKS, file number DRKS00006736, date registered September 20, 2014). Ethical approval for this study was obtained from the ethical review board of the medical faculty of the Technical University of Munich (file number 97/14). All procedures performed in studies involving human participants were in accordance with the ethical standards of the institutional and/or national research committee and with the 1964 Helsinki Declaration and its later amendments or comparable ethical standards. Written informed consent was obtained from all individual participants included in the study.

\section{Recruitment and participants}

As a result of advertisements in local media or local action days with the slogan "obesity", 271 interested persons contacted the local IHM teams for a provisional check of the feasibility for the study between October 2014 and February 2015. All persons who apparently complied with the requirements of the study were invited to a personal appointment with the trial physician to obtain full information and to undergo a comprehensive examination of the criteria for inclusion and exclusion.

Participants of both sexes aged 18-67 years with moderate overweight defined as BMI 28-35 were included in the study. Written informed consent was mandatory. Participants were not included if one of the following exclusion criteria was present: not legally competent, insufficient skills in German language, no private access to the Internet, already known pregnancy, known psychiatric disease, including eating disorder or addiction, known diseases of the eyes, known diabetes type 1 or 2 , hypertension $>$ grade I (systolic blood pressure $\geq 160 \mathrm{mmHg}$ or diastolic blood pressure $\geq 100 \mathrm{mmHg}$ ) with/ without medication, known heart disease, known gastric or 
duodenal ulcers, diseases of the liver or kidneys that do not allow an increased intake of proteins, disease-related impairments hampering certain elements of the lifestyle program, or therapeutic conditions not compatible with lifestyle modifications. According to the inclusion and exclusion criteria, 105 subjects were ineligible to be included in the study. The results of recruitment and enrollment of study participants are depicted in the consort study flowchart (Figure 1).

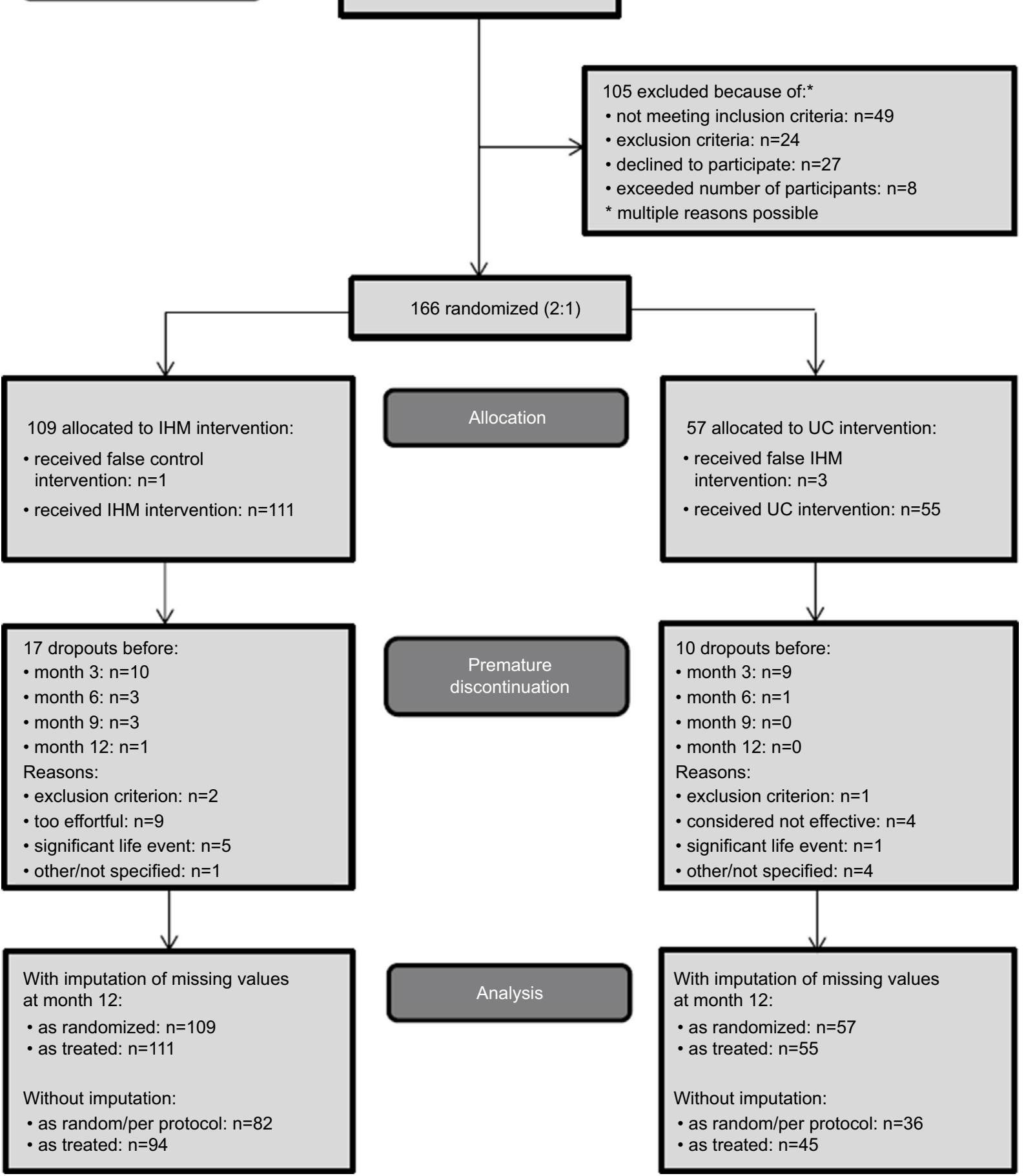

Figure I Consort flowchart showing the design of the study 


\section{Randomization}

One hundred and sixty-six eligible participants were randomized immediately after formal inclusion in the study. To enhance the willingness for study participation, we preferred a randomization ratio of 2:1: 109 subjects were allocated to the intervention group (IHM) and 57 to the control group (UC). Randomization and allocation envelopes were prepared by an independent statistician at the Institute for Medical Statistics and Epidemiology at the Technical University of Munich. The trial physicians were instructed to open the sealed envelopes in a strictly sequential order of enrollment and to disclose the allocated treatment arm to the study participant.

\section{Interventions}

The study compared two intervention arms - IHM and UC.

\section{IHM group}

The IHM lifestyle program, with an overall duration of 12 months, comprises two phases: 1) the first 3 months (reduction phase) include 3 full-day "introduction days" plus 10 2-hour weekly training sessions; and 2) during the following 9 months (maintenance phase), participants practice lifestyle modifications by themselves, supported by lifestyle counseling via telephone or email provided by trained IHM coaches. At 3-month intervals, they meet for a full-day refresher training session at the study center (Figure 2). The program encompasses access to a web-based health portal (www.viterio.de), providing detailed advice and instructions with respect to food, exercise, and relaxation. Furthermore, this tool allows personalized feedback control by written reports and graphics of the progress made. IHM was implemented as a "blended learning concept", with a combination of group interventions (group size of roughly 12), single face-to-face counseling, and an individual online portal. All training sessions were performed in the local study centers. Details of the intervention concept and realization are described elsewhere. ${ }^{15}$ With respect to the food component of the comprehensive lifestyle program, all participants were offered three different strategies for free choice: 1) calorie restriction day(s) (per week, 1 regular day, 5 waiver days, 1 calorie restriction day with $<900$ kcal plus fasting $<500 \mathrm{kcal}$ during week 7); 2) intermittent "fasting" (5 regular days, 2 serial calorie restriction days with $<600 \mathrm{kcal}$ for men and $<500 \mathrm{kcal}$ for women); and 3) meal replacement (1 regular day, 5 waiver days, one food restriction day with meal replacement $<900 \mathrm{kcal}$ plus meal replacement in week 7). All three strategies have proved to be successful in previous pilot groups.

\section{UC group}

This was an active control intervention that reflected common practice in UC with standard advice from a doctor. A leaflet containing 10 acknowledged rules for healthy food and physical exercise according to the German Nutrition Society was handed to the participants.

\section{Outcomes}

Body weight, height, waist circumference, blood pressure, and heart rates were measured by certified IHM coaches at each of the five examination visits. The teams were trained to perform the examinations in a standardized way (eg, subjects wearing light clothes and no shoes, with use of calibrated scales for measuring weight). Weight and waist circumference were measured twice and average values used in the analysis. On visits at months 0,3 , and
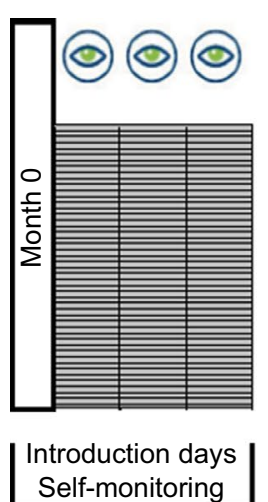

Introduction phase
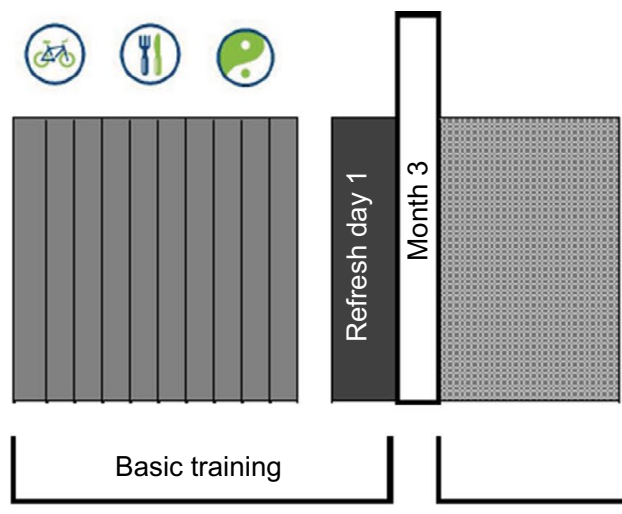

Reduction phase
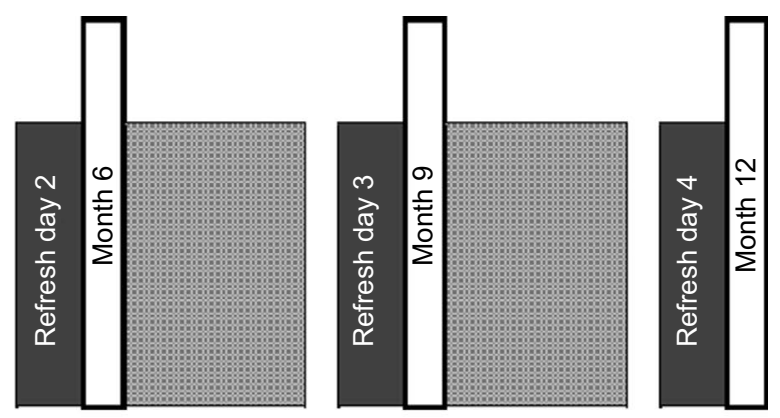

Distant lifestyle counseling

Maintaining phase

Figure 2 Schematic course of IHM in weight reduction with study examination visits embedded. Visits embedded (white bars). Introduction days (gray lined bars), $10 \times 2$-hour after-work sessions (gray bars) including self-monitoring $\odot$, physical activity $(-)$, nutrition $(\mathbb{D}$ and stress management packages $\odot$, and four quarterly refresh meetings (black bars).

Abbreviation: IHM, Individual Health Management. 
12 , blood samples were taken after overnight fasting in order to analyze glucose, serum lipids, and triglycerides as part of a standard laboratory examination. All other parameters were used for safety reasons. Body composition, which determines body fat in relation to muscle mass, was measured by bioimpedance analysis. All centers were equipped with a bioelectrical impedance analyzer (Forana; Helios, Frankfurt, Germany [Tethys software version 3.2]). At baseline examination, sociodemographic data such as education, employment status, and smoking and drinking habits were documented. The occurrence of adverse events (AEs) was captured systematically at each physical examination following the baseline test.

\section{Data analysis and statistical methods}

Weight reduction at month 12 was chosen a priori as the primary outcome variable to be used for hypothesis testing. Additionally, a $7.5 \%$ weight reduction at month 12 compared to baseline value was considered a responder and analyzed separately. Intermediate weight measures at months 3,6 , and 9 were analyzed secondarily.

The estimation of sample size was based on the assumption of a mean reduction in body weight after 12 months of $6 \mathrm{~kg}$ under IHM and of $3 \mathrm{~kg}$ under UC conditions. Data from the literature suggest a common standard deviation of $6 \mathrm{~kg}$ for change in weight. Therefore, the sample size for the $t$-test for independent groups ( $\alpha=0.05$, two-sided, power $80 \%$ ) was estimated as 98 in one group and 49 in the second (allocation ratio 2:1). Taking into account an expected dropout rate of $10 \%$, a total sample size of 160 participants was deemed appropriate.

Based on the intent-to-treat (ITT) population, the primary outcome (change in body weight $\left[\Delta_{\text {month 0-month } 12}\right]$ ) was tested using a general linear model with the grouping factors "intervention" and "study center", including the covariate baseline body weight. A 5\% level of significance (two-sided) was applied. In cases with missing data for weight at month 12 (dropouts), a conservative imputation technique was applied. Based on the available data for weight at months 0 and 12 , a linear regression equation was computed for each of the two intervention groups. Missing data in the intervention group were estimated by the terms of the regression from the control group and vice versa.

For additional sensitivity analyses, the imputation technique was modified in terms of a "realistic" imputation of missing data, meaning that missing data in one group were replaced by estimates derived from the regression of exactly this group. A further criterion for sensitivity analyses was to distinguish the ITT group from those subjects passing through the study "per protocol". Subjects were considered per protocol if 1 ) there existed no premature termination of the study, 2) there was no missing control examination, and 3) they were treated as randomized. Participants in the IHM group additionally had to adhere sufficiently to the intervention program, which means that they had to have attended at least 7 of the 10 group sessions during the first 3 months and missed not more than one refresher meeting at months 3-12.

Secondary end points were analyzed and compared by group as treated, without imputation of missing data. Differences with respect to baseline values and changes from month 0 to 12 between the two intervention groups were assessed by $t$-tests for independent samples. For differences between values at months 0 and $12, t$-tests for paired samples were applied in each of the two groups. All statistical tests were performed two-sided, with a significance level of 5\%, and in the sense of an exploratory approach. Therefore, no corrections for multiple testing were applied.

All captured data were analyzed descriptively by appropriate statistical parameters: absolute and relative frequencies for categorical data, and arithmetic means and standard deviations for numerical data. SPSS (version 22) was used for all statistical analyses. More details of the study protocol are available elsewhere. ${ }^{16}$

\section{Results \\ Sociodemographics and baseline characteristics}

In six of the study centers, the number of persons enrolled in the study varied from 18 to 25 . The remaining center brought in 37 participants, which necessitated the two IHM groups. Of the total of 166 persons, 109 were randomized to the intervention group (IHM) and 57 to the control group (UC). In the IHM and UC groups, 83 (76.1\%) and 40 (70.2\%) were female, respectively (between-group $P=0.457$ ). Mean age was 50.1 (SD 9.8) years in the IHM group and 51.6 (SD 9.9) years in the UC group $(P=0.367)$. These two groups were used for ITT hypothesis testing of the primary outcome body weight.

In four cases, subjects did not receive the treatment to which they were randomly allocated (see Figure 1). These four subjects received the other intervention by mistake, which was not suspected of being manipulated by trial physician or participant. As a consequence, 111 subjects received IHM and 55 were treated as usual. Comparable to the ITT group, age and gender showed no significant differences between the 
Table I Basic sample description and baseline values for secondary outcome parameters

\begin{tabular}{|c|c|c|c|c|c|}
\hline \multirow[t]{2}{*}{ Baseline variable } & \multicolumn{2}{|c|}{ IHM $(n=I I I)$} & \multicolumn{2}{|c|}{ UC $(n=55)$} & \multirow[t]{2}{*}{$P$} \\
\hline & Mean & $\overline{S D}$ & Mean & $\overline{\text { SD }}$ & \\
\hline \multirow[t]{2}{*}{ Age, years } & 49.9 & 9.7 & 52.1 & 10 & 0.182 \\
\hline & $n$ & $\%$ & $\mathbf{n}$ & $\%$ & \\
\hline Female & 83 & 74.8 & 40 & 72.7 & 0.851 \\
\hline \multicolumn{6}{|l|}{ Education } \\
\hline Primary school & 29 & 26.1 & 14 & 25.5 & 0.555 \\
\hline Secondary school & 55 & 49.5 & 26 & 47.3 & \\
\hline High school & 27 & 24.3 & 14 & 25.5 & \\
\hline Other & 0 & & I & 1.8 & \\
\hline \multicolumn{6}{|l|}{ Employment status } \\
\hline Employed & 90 & 81.1 & 44 & 80 & 0.392 \\
\hline Occupied without payment & 6 & 5.4 & I & 1.8 & \\
\hline Jobless & 2 & 1.8 & I & 1.8 & \\
\hline Still in education & 0 & & 1 & 1.8 & \\
\hline Retired & 13 & 11.7 & 8 & 14.5 & \\
\hline \multicolumn{6}{|l|}{ Living condition } \\
\hline Single household & 10 & 9.1 & 7 & 13 & 0.43 \\
\hline Multiperson household & 100 & 90.9 & 47 & 87 & \\
\hline \multicolumn{6}{|l|}{ Smoker } \\
\hline No & 98 & 88.3 & 50 & 90.9 & 0.792 \\
\hline Yes & 13 & 11.7 & 5 & 9.1 & \\
\hline - If yes, $<5$ cigarettes per day & 3 & & 1 & & \\
\hline - If yes, 5-30 cigarettes per day & 10 & & 4 & & \\
\hline \multicolumn{6}{|l|}{ Alcohol consumption } \\
\hline No & 61 & 55 & 24 & 43.6 & 0.189 \\
\hline Yes & 50 & 45 & 31 & 56.4 & \\
\hline - If yes, <I glass per day & 34 & & 21 & & \\
\hline - If yes, I-2 glasses per day & 10 & & 8 & & \\
\hline \multirow[t]{2}{*}{ - If yes, 3-4 glasses per day } & 1 & & 0 & & \\
\hline & Mean & SD & Mean & SD & $P$ \\
\hline Body weight, kg & 89.6 & 10.2 & 88.9 & 11.3 & 0.681 \\
\hline BMI & 31.8 & 2 & 31.5 & 2 & 0.48 \\
\hline Waist circumference, $\mathrm{cm}$ & 103.7 & 8.8 & 103.2 & 9.9 & 0.743 \\
\hline Systolic blood pressure, $\mathrm{mmHg}$ & $|3| .4$ & 15.8 & 134.1 & 16.7 & 0.314 \\
\hline Diastolic blood pressure, $\mathrm{mmHg}$ & 84.3 & 8.4 & 85.6 & 8.7 & 0.377 \\
\hline Heart rate, beats per min & 72.7 & 10.7 & 74.7 & 10.2 & 0.244 \\
\hline BF, kg & 35.5 & 6.7 & 35 & 5.1 & 0.629 \\
\hline BCM, kg & 27.1 & 5.6 & 26.9 & 6.1 & 0.813 \\
\hline BF:BCM ratio & 1.37 & 0.35 & 1.36 & 0.32 & 0.948 \\
\hline Fasting glucose, $\mathrm{mg} / \mathrm{dL}$ & 92.5 & 12.9 & 92.6 & 11.8 & 0.959 \\
\hline Total cholesterol, mg/dL & 227.6 & 38 & 231.1 & 39.7 & 0.585 \\
\hline LDL cholesterol, mg/dL & 149.3 & 33.7 & 150.6 & 31.3 & 0.813 \\
\hline HDL cholesterol, $\mathrm{mg} / \mathrm{dL}$ & 58.3 & 13.3 & 59.6 & 15.9 & 0.587 \\
\hline LDL:HDL ratio & 2.73 & 0.98 & 2.71 & 0.9 & 0.868 \\
\hline Triglycerides, $\mathrm{mg} / \mathrm{dL}$ & 137 & 76.8 & 134.5 & 61.8 & 0.832 \\
\hline
\end{tabular}

Note: Group allocation "as treated".

Abbreviations: IHM, Individual Health Management; UC, usual care; SD, standard deviation; BF, body fat; BCM, body cell mass; BMI, body mass index; LDL, low-density lipid; HDL, high-density lipid.

groups (Table 1). With respect to education, employment status, and smoking and drinking habits, there were no observed significant differences between the study groups (Table 1). Further, secondary outcome parameters with means and standard deviations for both study groups are also presented in Table 1. Mean BMI was 31.8 (SD 2) for the IHM group and 31.5 (SD 2) for the UC group. There were no statistically significant differences between the groups in respect of weight and waist circumference, blood pressure, body fat, muscle mass, or laboratory findings (Table 1). 


\section{Dropout and protocol violations}

As depicted in the consort study flowchart (Figure 1), both study groups saw attrition during the 12-month study. Of 111 subjects who commenced with the IHM group, 17 (15.3\%) prematurely discontinued the study, while in the UC group the dropout rate was $18.2 \%$ (10 of 55). The majority of dropouts left the study before the control visit at month 3 , and this occurred in 10 of 17 cases in the IHM group and in 9 of 10 in the UC group. The most frequent reasons were "overburdened" in the IHM group and "considered not effective" in the UC group.

The defined preconditions of passing through the whole study "per protocol" were fulfilled by 82 and 36 participants in the IHM and UC groups, respectively. Those four cases with false treatment allocation were not considered per protocol. Of the participants of the IHM group with available data at month 3, 68\% showed $100 \%$ adherence to the intervention during the first 3 months (participation in two introduction days and in all of the 10 after-work meetings). Participation rates for the quarterly refresh meetings were $89 \%-96 \%$ of the study participants at that time.

\section{Body weight}

Based on the ITT population with conservative imputation of missing values, the main outcome parameter of body weight showed a mean decrease of $8.7 \mathrm{~kg}$ (SD 6.1, $\mathrm{n}=109$ ) in the intervention group (IHM) and $4.2 \mathrm{~kg}$ (SD $5, \mathrm{n}=57$ ) in the control group (UC) at month 12. Weight at baseline was $89.5 \mathrm{~kg}$ (SD 10.3) in the IHM group and $89.2 \mathrm{~kg}$ (SD 11.1) in the UC group. General linear model testing of the change values revealed a highly significant difference between the groups $(P<0.001)$. Neither the factor "study center" nor the interaction "center $\times$ group" were statistically significant $(P=0.101$ and $P=0.253$, respectively). The covariate of baseline weight showed a statistically significant impact $(P=0.033)$. Therefore, the primary null hypothesis of no differences between the groups in weight change had to be rejected. Additionally, it was shown that in both groups, each weight reduction from month 0 to 12 was statistically significant $(P<0.001$, $t$-test for paired groups).

In the per protocol population, the mean weight reduction was $10.1 \mathrm{~kg}$ (SD 6.2, $\mathrm{n}=82)$ in the IHM group and $3 \mathrm{~kg}$ (SD 3.7, $\mathrm{n}=36$ ) in the UC group. The mean weight reduction from baseline to month 12 was $11 \%$ and $2.7 \%$ for IHM and $\mathrm{UC}$, respectively. The respective figures were $9.9 \mathrm{~kg}$ (SD 5.5, $\mathrm{n}=111)$ and $2.5 \mathrm{~kg}$ (SD 3.5, n=55) when groups were analyzed as treated with realistic imputation of missing values at month 12. Without imputation, mean weight reduction was $9.9 \mathrm{~kg}$ (SD 6, n=94) in the IHM group and $2.4 \mathrm{~kg}$ (SD $3.9, \mathrm{n}=45$ ) in the control group (UC). All three sensitivity analyses confirmed a statistically significant effect between the groups $(P<0.001)$, while showing no significant effects of either the factor "center" nor the interaction term "center $\times$ group".

The mean course of the quarterly measurements of weight is depicted in Figure 3. To avoid the possible impact on mean values due to attrition during the study period, only subjects with complete data sets (per protocol) were used for this analysis. For each sex, the graph shows the most distinct weight reduction during the first 3 months, followed by a nearly horizontal line, indicating no weight gain toward the end of the study. The differences between both intervention groups are more pronounced in men.

Sixty-two out of $94(66.0 \%)$ participants in the IHM group showed a weight reduction of at least $7.5 \%$ of baseline value at month 12 , while this response criterion was fulfilled by only 6 of $45(13.3 \%)$ participants in the UC group. This difference proved to be statistically significant $(P<0.001$, Fisher's exact test). Displaying all individual pairs of measurements in a scatterplot, it can be demonstrated that beneficial weight reductions in the IHM group were distributed equally across the whole range of baseline measures (Figure 4).

The preferred nutritional regimen was fasting in 34\%, 2 -day diet in $37 \%$, and meal replacement in $14 \%$ of the IHM participants; $15 \%$ showed a changing diet pattern over time. Weight reduction at month 12 was similar in all subgroups, presenting mean values of $10.6 \mathrm{~kg}$ (SD 6.6), $10.3 \mathrm{~kg}$ (SD 5.4), $7.4 \mathrm{~kg}$ (SD 5.5), and $9.3 \mathrm{~kg}$ (SD 6.3), respectively.

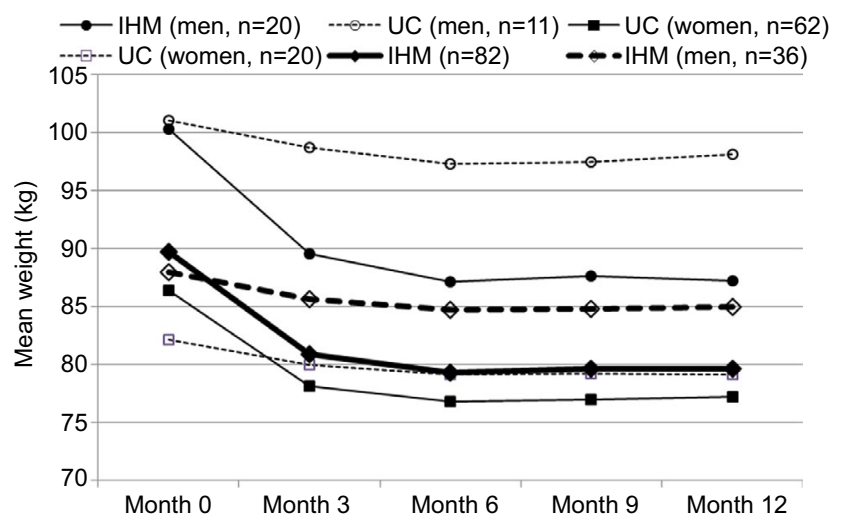

Figure 3 Mean weight in both treatment groups during the course of the study, separated by gender.

Notes: Thick black lines represent whole-group totals; per protocol subjects only Abbreviations: IHM, Individual Health Management; UC, usual care. 


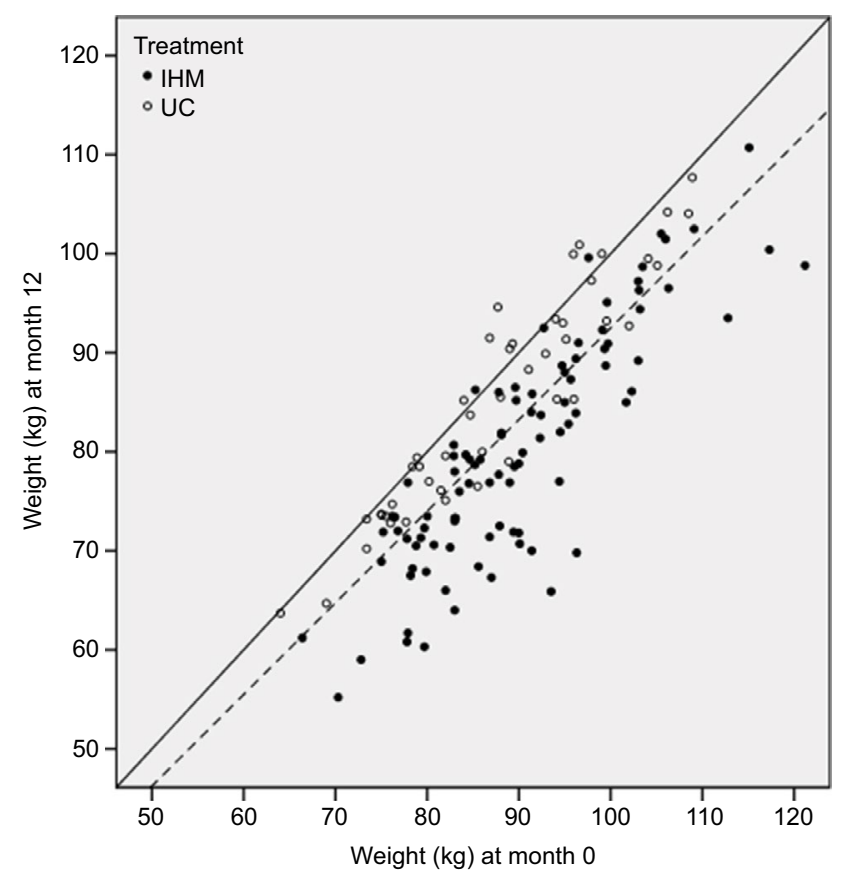

Figure 4 Scatterplot for weight measurements at months 0 and 12 (without imputations).

Notes: Solid line shows diagonal of equal values at both examinations; dashed line represents a $7.5 \%$ reduction at month 12 .

Abbreviations: IHM, Individual Health Management; UC, usual care.

\section{Secondary outcome variables}

Table 2 shows the mean changes from month 0 to 12 per group for all secondary outcomes. In the IHM group, all parameters improved significantly, except total cholesterol. Under control intervention, only BMI, waist circumference, body fat, and ratio of fat and body cell mass presented significant changes. However, with respect to these parameters, the differences between the groups proved to be significantly higher in the IHM group. In addition, the mean increase in high-density lipid cholesterol was significantly higher under IHM intervention compared to the control intervention. Furthermore, changes in blood pressure, fasting glucose, cholesterol, and triglycerides in the IHM group were superior to the control group, but failed to reach statistical significance. In addition, body composition was analyzed separately for men and women. While men showed significantly higher means for muscle cell mass at baseline, there were no significant interaction effects between treatment and sex with respect to pre-post changes in parameters of the bioimpedance analysis.

\section{Adverse events}

For 101 participants of the IHM group and 46 of the UC group, at least one control visit (months 3-12) was carried out and documented. Therefore, these groups served as the basis for the analysis of AEs. In 36 (35.6\%) subjects of the IHM group, at least one $\mathrm{AE}$ was documented during the study, of which 17 were graded as "slight", 19 as "moderate", and eight as "strong". In 23 of these participants, no causal relation to the study intervention was given. In seven cases, a relation was considered "possible" and "probable" in eight cases. Of the control group, in nine (19.6\%) subjects at least one AE was documented (one slight, five moderate, and four strong), with only one having probable relation to the intervention. Under IHM intervention with at least possible

Table 2 Secondary outcome parameter: change from baseline to month 12

\begin{tabular}{|c|c|c|c|c|c|}
\hline \multirow[t]{2}{*}{ Secondary outcome variable } & \multicolumn{2}{|c|}{ IHM $(n=94)$} & \multicolumn{2}{|c|}{ UC $(n=45)$} & \multirow{2}{*}{$\begin{array}{l}\text { Between-group } \\
\text { difference } \\
P \\
\end{array}$} \\
\hline & Mean & SD & Mean & SD & \\
\hline BMI & $-3.5 * *$ & 2.15 & $-0.87^{* *}$ & 1.36 & $<0.001$ \\
\hline Waist circumference, $\mathrm{cm}$ & $-11.75 * *$ & 7.58 & $-5.26 * *$ & 5.27 & $<0.001$ \\
\hline Systolic blood pressure, $\mathrm{mmHg}$ & $-5.23 * *$ & 17.28 & 0.82 & 17.67 & 0.057 \\
\hline Diastolic blood pressure, $\mathrm{mmHg}$ & $-3.23 * *$ & 9.41 & -1.11 & 11 & $0.24 I$ \\
\hline Heart rate & $-2.54 *$ & 11.19 & -2.84 & 10.09 & 0.878 \\
\hline BF, kg & $-6.73 * *$ & $5.34^{\#}$ & $-1.7 \mid * *$ & 3.59 & $<0.001$ \\
\hline BCM, kg & $-1.84 * *$ & 1.7 & -0.55 & 2.16 & $<0.001$ \\
\hline BF:BCM ratio & $-0.18^{* *}$ & 0.19 & $-0.05^{*}$ & 0.17 & $<0.001$ \\
\hline Fasting glucose, $\mathrm{mg} / \mathrm{dL}$ & $-4.8 * *$ & $13.45^{\S}$ & -1.42 & 12.34 & 0.16 \\
\hline Total cholesterol, mg/dL & -2.4 & $28.28^{\#}$ & 0.6 & 35.81 & 0.594 \\
\hline LDL cholesterol, $\mathrm{mg} / \mathrm{dL}$ & $-7.07^{* *}$ & $24.10^{\#}$ & -2.62 & 30.06 & 0.351 \\
\hline HDL cholesterol, mg/dL & $5.04 * *$ & $9.19^{\#}$ & 1.6 & 7.8 & 0.032 \\
\hline LDL:HDL ratio & $-0.32^{* *}$ & $0.59^{\#}$ & -0.14 & 0.52 & 0.081 \\
\hline Triglycerides, mg/dL & $-18.44^{*}$ & $70.8 I^{\#}$ & 1.7 & 79.34 & 0.135 \\
\hline
\end{tabular}

Notes: Within group difference between baseline and month 12 : ${ }^{*}<<0.05 ; * P<0.01 ;{ }^{*} n=93 ;{ }^{\S} n=90$. Group allocation as treated, without imputation of missing values.

Abbreviations: IHM, Individual Health Management; UC, usual care; SD, standard deviation; BF, body fat; BCM, body cell mass; BMI, body mass index; LDL, low-density lipid; HDL, high-density lipid. 
causality and moderate degree of severity, the following AEs were documented: nausea, headache or bad-tempered during fasting, panic attacks, joint pain, dry skin, and hair loss. All symptoms had completely resolved by the end of the study. Three AEs in the IHM group and one in the UC group were classified as "severe". In all, causality by the study intervention was excluded.

\section{Discussion}

IHM as a 1-year lifestyle program has clearly proved to be superior to standard advice from the doctor in terms of weight reduction in people with BMI of 28-35. Based on the ITT analysis, a mean decrease of $8.7 \mathrm{~kg}$ at month 12 was observed in the IHM group, while under UC conditions, the mean weight reduction was $4.2 \mathrm{~kg}$. This highly significant difference $(P<0.001)$ was achieved, although the primary analysis followed a "conservative" approach, implying that the imputation technique of missing values, as well as the handling of false treatment allocations, favored the control group. All sensitivity analyses confirmed the result of the primary hypothesis testing, just like that of the subgroup of participants who provided complete data sets and sufficient adherence to the lifestyle program, showing weight reductions of $10.1 \mathrm{~kg}$ and $3 \mathrm{~kg}$ for the IHM and UC groups, respectively, and thus presenting a more distinct betweengroup difference.

The analysis of this multicenter study did not reveal any significant center effect, implying that the beneficial effects of IHM were similar in all study centers, which is considered a sign of robustness of the main study results. As such, it does not suggest doubts regarding the generalizability of the results. Based on a structured IHM concept and also provided there exists standardized high-quality educational training of the IHM coaches, similar results may be expected in other settings.

Research has shown that overweight individuals are successful at long-term weight loss when defined as losing at least $5 \%-10 \%$ of initial body weight and maintaining the loss for at least 1 year. ${ }^{7,17}$ Guidelines for the management of overweight and obesity in adults from the American Heart Association suggest that high-intensity comprehensive lifestyle programs should achieve average weight losses of approximately $8 \mathrm{~kg}$ in a 6-month period. ${ }^{7}$ Christian et $\mathrm{al}^{18}$ pooled the success rates of various "intense" lifestyle interventions (13-52 hours of care) and reported weight loss of $\geq 10 \%$ in $28 \%, 5 \%-9.9 \%$ in $26 \%, 0-4.9 \%$ in $38 \%$, and no loss/ weight gain in $26 \%$ of the participants (percentages do not add up to $100 \%$ due to the pooling algorithm). When these categories are applied to the data of our IHM group, percentages of $49 \%, 34 \%, 15 \%$, and $2 \%$ are derived, respectively.

Obesity is a lifelong challenge for all persons concerned. Beyond weight reduction, maintaining weight loss and keeping a healthy weight are the important issues that have to be solved from the long-term perspective. In contrast to usual patterns of weight loss in people undergoing a lifestyle intervention who achieve maximum weight loss at 6 months, the graph of our mean weight measurement did not show subsequent weight gain over time, but held a plateau in the following course of mean weight. ${ }^{17,19}$ This program adherence might have been due to the individual web-based health portal, with some tools for self-monitoring and performance measures in order to support participants to monitor weight and physical activity continually, and self-reports during their 1 -year program. Findings from the literature indicate that daily self-weighing and self-monitoring by a pedometer are associated with more distinct weight reduction and decreased risk of weight regain. ${ }^{7,14}$

IHM offered different forms of diet for free choice. The most popular nutritional regimens in the present study were 2-day diet and fasting. However, there were no statistically significant differences between mean weight reductions. All subgroups with respect to diet form were approximately equally effective on weight reduction. ${ }^{20}$ These results are not really surprising, because it is known from other studies that in programs for weight reduction, it is more important to serve with effective support to adhere to lifestyle changes than to provide reputed highly attractive procedures (different types of diet). ${ }^{13,21}$

IHM was also more beneficial in the reduction of blood pressure, although just failing statistical significance. Similar findings were observed in reduction of fasting glucose and serum lipids. Increase in high-density lipid cholesterol was statistically significant and superior in the IHM group compared to the UC group. Bioimpedance analysis showed a more pronounced reduction in body fat after IHM, indicating that reduction in body weight was mainly due to decreased body fat. ${ }^{22}$ Unfortunately, weight loss was also associated with a decrease in muscle cell mass. However, the more pronounced decrement of the ratio of body fat and muscle cell mass under IHM elucidates that the reduction in fat outweighs the muscle-lowering effect. These beneficial findings for IHM were observed similarly in both sexes. However, more emphasis should be put on muscle-building exercises. ${ }^{23}$ Lifestyle modification is a key issue with regard to risk reduction for diabetes, hypertension, and metabolic syndrome. ${ }^{24,25}$ For such diseases, the positive findings of the 
TALENT study on weight reduction suggest a preventive benefit of IHM.

\section{Strengths and limitations}

The study was monitored by a professional institution with acknowledged expertise in supervision of clinical trials. The monitoring plan comprised central indoor monitoring and onsite visits at the participating study centers. The monitoring reports revealed no serious issues in the on-site conduction of the study.

Dropout rates in the IHM and UC groups of $15 \%$ and $18 \%$, respectively, and the fact that about $71 \%$ of all study participants fulfilled the per protocol criteria demonstrate the difficulties in long-term lifestyle intervention studies. Nevertheless, our dropout rate was lower than most other clinical studies in this field (eg, Weight Watchers - 39\%). ${ }^{26}$ Although several measures were undertaken to reduce attrition, some IHM participants discontinued the program. Not all IHM participants were able to attend all group meetings, due to individual requirements of daily life. The variety of all these different aspects corresponds to the tenets of IHM as an "individualized" way of health management, and thus not categorized as experimental bias. In light of these challenges, the study was able to accomplish credible and robust findings.

Based on a blended learning concept and using web-based e-health tools, the program could provide participants with a comprehensive long-term management of overweight and obesity and its complications for everyday life over a period of at least 1 year. Furthermore, the multicenter study could demonstrate that intensive and comprehensive lifestyle training can be implemented successfully in local health and prophylaxis centers at various health resorts, in order to improve the medical quality of their health services.

\section{Author contributions}

DM was the principal investigator of the study and developed the lifestyle program IHM. PL and EW contributed to implementation and realization of IHM in two of the spas. VK was responsible for the concept and conduct of the confirmatory statistical analysis. WW participated in the conception and data management of the trial and drafted the manuscript. All authors contributed toward data analysis, drafting and critically revising the paper, gave final approval of the version to be published, and agree to be accountable for all aspects of the work.

\section{Acknowledgments}

We would like to thank the members of the local IHM campus teams at the following participating Bavarian spas for their cooperation and support: Bad Alexandersbad, Bad Griesbach, Bad Kötzting, Bad Tölz, spa town of Treuchtlingen, and Bad Wörishofen; and in particular the study physicians Arnulf Bultmann, Wolfgang Doerfler, Axel Eustachi, Stefan Hager, Charlotte Pfitzner, Nicole Reinelt, Cordula von der Ropp, and Guido Weixel. In addition, our thanks go to Alexander Hapfelmeier, Institute for Medical Statistics and Epidemiology, Technische Universität München, for providing the randomization list. The study received funding from the Bavarian State Ministry of Health and Care, forwarded by the participating spas, which obtained financial support from a funding program in order to enhance medical quality (grants K1-04-00004 to 9-2012-EA_BayGA).

\section{Disclosure}

The authors report no conflicts of interest in this work.

\section{References}

1. NCD Risk Factor Collaboration. Trends in adult body-mass index in 200 countries from 1975 to 2014 : a pooled analysis of 1698 populationbased measurement studies with 19.2 million participants. Lancet. 2016;387:1377-1396.

2. Main ML, Rao SC, O'Keefe JH. Trends in obesity and extreme obesity among US adults. JAMA. 2010;303:1695-1696.

3. Global BMI Mortality Collaboration. Body-mass index and all-cause mortality: individual-participant-data meta-analysis of 239 prospective studies in four continents. Lancet. 2016;388:776-786.

4. Flegal KM, Kit BK, Orpana H, Graubard BI. Association of all-cause mortality with overweight and obesity using standard body mass index categories: a systematic review and meta-analysis. JAMA. 2013;309:71-82.

5. Webber L, Divajeva D, Marsh T, et al. The future burden of obesity related diseases in the 53 WHO European-region countries and the impact of effective interventions: a modelling study. BMJ Open. 2014;4:e004787.

6. Fontana L, Hu FB. Optimal body weight for health and longevity: bridging basic, clinical, and population research. Aging Cell. 2014;13:391-400.

7. Jensen MD, Ryan DH, Apovian CM, et al. 2013 AHA/ACC/TOS guideline for the management of overweight and obesity in adults: a report of the American College of Cardiology/American Heart Association Task Force on Practice Guidelines and the Obesity Society. Circulation. 2014;129:S102-S138.

8. Dietz WH, Baur LA, Hall K, et al. Management of obesity: improvement of health-care training and systems for prevention and care. Lancet. 2015;385:2521-2533.

9. Aveyard P, Lewis A, Tearne S, et al. Screening and brief intervention for obesity in primary care: a parallel, two-arm, randomised trial. Lancet. 2016;388:2492-2500.

10. Wadden TA, Volger S, Sarwer DB, et al. A two-year randomized trial of obesity treatment in primary care practice. $N$ Engl J Med. 2011;365:1969-1979.

11. Maruthur N, Gudzune K, Hutfless S, et al. Avoiding weight gain in cardiometabolic disease: a systematic review. JObes. 2014;2014:358919.

12. Atallah R, Filion KB, Wakil SM, et al. Long-term effects of 4 popular diets on weight loss and cardiovascular risk factors: a systematic review of randomized controlled trials. Circ Cardiovasc Qual Outcomes. 2014;7:815-827.

13. Wirth A, Wabitsch M, Hauner H. The prevention and treatment of obesity. Dtsch Arztebl Int. 2014;111:705-713.

14. Soleymani T, Daniel S and Garvey WT. Weight maintenance: challenges, tools and strategies for primary care physicians. Obesity rev. 2016;17:81-93. 
15. Melchart D, Eustachi A, Wellenhofer-Li Y, Doerfler W, Bohnes E. Individual health management: a comprehensive lifestyle counselling programme for health promotion, disease prevention and patient education. Forsch Komplementmed. 2016;23:30-35.

16. Melchart D, Doerfler W, Eustachi A, Wellenhofer-Li Y, Weidenhammer W. The Talent study: a multicentre randomized controlled trial assessing the impact of a "tailored lifestyle self-management intervention" (talent) on weight reduction. BMC Obes. 2015;2:38.

17. Montesi L, Ghoch ME, Brodosi L, Calugi S, Marchesini G, Grave RD. Long-term weight loss maintenance for obesity: a multidisciplinary approach. Diabetes Metab Syndr Obes. 2016;9:37-46.

18. Christian JG, Tsai AG, Bessesen DH. Interpreting weight losses from lifestyle modification trials: using categorical data. Int J Obes. 2010;34:207-209.

19. ter Bogt NC, Bemelmans WJ, Beltman FW, Broer J, Smit AJ, van der Meer K. Preventing weight gain: one-year results of a randomized lifestyle intervention. Am J Prev Med. 2009;37:270-277.

20. Gardner CD, Kiazand A, Alhassan S, et al. Comparison of the Atkins, Zone, Ornish, and LEARN diets for change in weight and related risk factors among overweight premenopausal women: the A TO Z Weight Loss Study: a randomized trial. JAMA. 2007;297:969-977.
21. Taylor RW, Roy M, Jospe MR, et al. Determining how best to support overweight adults to adhere to lifestyle change: protocol for the SWIFT study. BMC Public Health. 2015;15:861.

22. Aslam M, Eckhauser AW, Dorminy CA, Dossett CM, Choi L, Buchowski MS. Assessing body fat changes during moderate weight loss with anthropometry and bioelectrical impedance. Obesity Res Clin Pract. 2009;3:209.

23. Metcalf B, Rabkin RA, Rabkin JM, Metcalf LJ, Lehman-Becker LB. Weight loss composition: the effects of exercise following obesity surgery as measured by bioelectrical impedance analysis. Obes Surg. 2005;15:183-186.

24. Wennehorst K, Mildenstein K, Saliger B, et al. A comprehensive lifestyle intervention to prevent type 2 diabetes and cardiovascular diseases: the German CHIP trial. Prev Sci. 2016;17:386-397.

25. Grave RD, Calugi S, Centis E, Marzocchi R, El Ghoch M, Marchesini G. Lifestyle modification in the management of the metabolic syndrome: achievements and challenges. Diabetes Metab Syndr Obes. 2010;3:373-385.

26. Jebb SA, Ahern AL, Olson AD, et al. Primary care referral to a commercial provider for weight loss treatment versus standard care: a randomised controlled trial. Lancet. 2011;378:1485-1492.
Diabetes, Metabolic Syndrome and Obesity: Targets and Therapy is an international, peer-reviewed open-access journal committed to the rapid publication of the latest laboratory and clinical findings in the fields of diabetes, metabolic syndrome and obesity research. Original research, review, case reports, hypothesis formation, expert opinion and commentaries are all considered for publication. The manuscript management system is completely online and includes a very quick and fair peer-review system, which is all easy to use. Visit http://www.dovepress.com/testimonials.php to read real quotes from published authors. 